\title{
Star Formation Along the Bars of Virgo Cluster and Isolated Spiral Galaxies
}

\author{
Rebecca A. Koopmann and Jeffrey D. P. Kenney \\ Astronomy Department, Yale University, New Haven, CT 06520-8101
}

\section{Introduction}

Star formation along the bar is common in late-type barred galaxies (Phillips and Garcia-Barreto et al., these proceedings), which tend to have weak stellar bars. It is rarer in strongly barred galaxies, e.g., early-type spiral galaxies. Abundance gradient measurements (Martin \& Roy 1995) and simulations (Friedli \& Benz 1995) suggest that star formation along the bar of strongly barred galaxies may indicate a young bar. Since bars can be created in tidal interactions and mergers (Noguchi 1987), it is of interest to investigate the star formation properties of barred galaxies in different environments. We use $\mathrm{H} \alpha$ images to compare the star formation properties in the bar region of a sample of barred galaxies in the Virgo Cluster and a control sample of isolated galaxies. We focus on the three galaxies with the strongest star formation along the bar, including NGC 4424, a possible merger remnant.

\section{The Sample of Barred Spiral Galaxies}

The Virgo Cluster sample contains spiral galaxies with inclinations between 30 and 70 degrees with $\mathrm{B}_{T} \leq 13$ and is complete to $\mathrm{B}_{T}=12$. The isolated sample was selected from the Tully Nearby Galaxies catalog and an isolated galaxy list provided by P. Fouqué (private communication). Bar type was not a selection criterion for either sample. Sample properties are:

\begin{tabular}{|c|c|c|c|}
\hline & & Isolated & Virgo \\
\hline & Sample Si & 34 & 55 \\
\hline & & $50 \%$ & $25 \%$ \\
\hline & $S$ & $76 \%$ & $56 \%$ \\
\hline . & $\operatorname{tar}$ & $27 \%(7 / 26)$ & $29 \%(9 / 31)$ \\
\hline
\end{tabular}

\section{Star Formation along the Bar}

We find HII regions along the bar in 7 isolated galaxies and 9 Virgo Cluster galaxies. Histograms of the $\mathrm{H} \alpha$ luminosity along the bar (excluding any central emission) is shown for our two samples in Figure 1. Although the fraction of galaxies with star formation along the bar is similar in both samples, there is a tendency for the $\mathrm{H} \alpha$ luminosity along the bar to be higher in Virgo Cluster galaxies. This may not be significant, since our sample size is small. The apparent difference between the samples at low luminosities does not appear to be caused by a distance effect. All of the galaxies with star formation along the bar are of type SBbc or later, except four Virgo Cluster galaxies, which are 


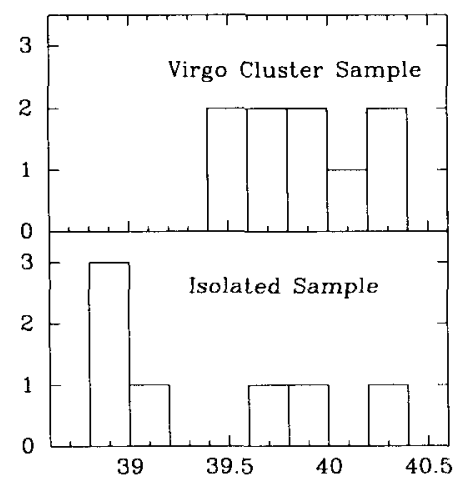

Figure 1. Histograms of the $\log$ of $\mathrm{H} \alpha$ luminosity $\left(\mathrm{erg} \mathrm{s}^{-1}\right)$ along the bar in isolated and Virgo Cluster galaxies.

classified in the RC3 as SBa - SBab. These include NGC 4064 and NGC 4424. $R$ and $\mathrm{H} \alpha$ images of NGC 4424, NGC 4064, and NGC 3359, the galaxies with the highest $\mathrm{H} \alpha$ luminosity along the bar in our sample, are shown in Figure 2.

\subsection{NGC 3359}

NGC 3359 is classified as SB(rs)c II in the RC3 and SBc(s)I.8 pec in the RSA. It has a strong spiral arm to the south with a weaker counterpart and several flocculent arms to the north. It is strongly barred with a bar strength more similar to a typical Sa galaxy than a typical Sc galaxy (Martin 1995). Five bright HII regions, which contribute $13 \%$ of the $\mathrm{H} \alpha$ flux of the galaxy, lie along the bar. Martin \& Roy (1995) estimate that the age of the bar is $<1$ Gyr, based on the $\mathrm{O} / \mathrm{H}$ abundance gradient across the disk. There is no direct evidence of a tidal interaction or minor merger. NGC 3359 may have a faint companion (Ball 1986), but it is probably too small to explain NGC 3359's morphology.

\subsection{NGC 4064 and NGC 4424}

The most unusual galaxies in the Virgo Cluster sample are NGC 4064 and NGC 4424 , which have $\mathrm{H} \alpha$ morphologies unlike any of the isolated galaxies. All of the star formation in these galaxies is concentrated in a nearly linear ridge of bright HII regions within the central $1 \mathrm{kpc}$. This circumnuclear emission is enhanced with respect to both early- and late-type Virgo Cluster spirals. Classification of these galaxies is uncertain because they have small bulges, but little structure and no star formation in the outer disks. This type of galaxy may be unique to cluster environments. The RC3 classifies both NGC 4424 and NGC 4064 as SBa:, while the RSA classifies NGC 4424 as S(a?)pec and NGC 4064 as SBc(s):. The galaxies are located in very different environments within the Virgo Cluster. NGC 4424 is in a dense region, 1 degree from the center of subcluster $B$ around M49, while NGC 4064 is located in the lower density outskirts of the Virgo Cluster. We believe that NGC 4424 is one of the clearest cases among spiral galaxies in the Virgo Cluster for a significant ( $\sim 0.1-0.5$ mass ratio) and recent 

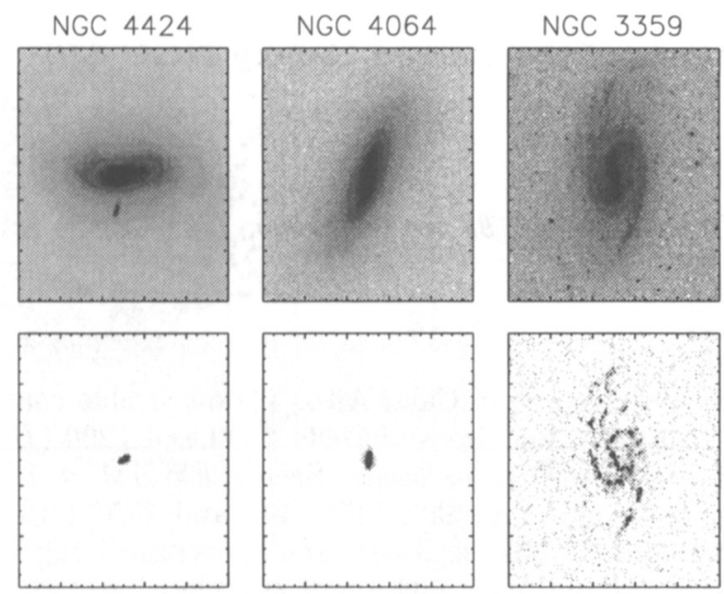

Figure 2. $R$ (top) and $\mathrm{H} \alpha$ (bottom) images of NGC 4424, NGC 4064, and NGC 3359.

merger (Kenney et al. 1995). The $R$-band image shows broad shell-like features in the outer disk, isophote twisting, and unusual banana-shaped isophotes at intermediate radii. Optical spectra at four different position angles, including the isophotal major axis, show extremely low line-of-sight velocities, perhaps consistent with bar streaming motions. The merger may be responsible for the creation of the bar, as is seen in minor merger simulations (Mihos \& Hernquist 1994). The banana-shaped distribution may be the result of a merger-induced bending instability (Hernquist et al. 1993). The elliptical galaxy near NGC 4424 has an unknown redshift, but even if it is at the same distance, it is probably too small to have caused the disturbances, unless it is the core of a larger galaxy which is merging with NGC 4424. The history of NGC 4064 is less clear. The galaxy is highly inclined, so it is harder to see structure in the outer disk. The dominant structure is the bright central bar, which connects smoothly with loosely wound spiral arms. There are no obvious companions.

\section{References}

Ball, R. 1986, ApJ, 307, 453

Friedli, D. \& Benz, W. 1995, A\&A, 301, 649

Hernquist, L., Heyl, J. S., \& Spergel, D. N. 1993, ApJ, 416, L9

Kenney, J. D. P., Koopmann, R. A., Rubin, V. C., \& Young, J. S. 1995, AJ, in press

Martin, P. 1995, AJ, 109, 2428

Martin, P. \& Roy, J.-R. 1995, ApJ, 445, 161

Mihos, J. C. \& Hernquist, L. 1994, ApJ, 425, L13

Noguchi, M. 1987, MNRAS, 228, 635 